\title{
REVIEW ARTICLE \\ Heterogeneity of mesenchymal stem cells: characterization and application in cell therapy
}

\author{
Xingzhi Liul,2†, Zhihua Zhao ${ }^{2 \dagger}$, Zhe Zhao ${ }^{2}$, Zhongjuan Xu',2, Junjun $\mathrm{Cao}^{3}$, Bin Wang ${ }^{4}$ and \\ Guangli Suo 2* \\ ISchool of Nano-Tech and Nano-Bionics, University of Science and Technology of China, Hefei, Anhui, China; \\ ${ }^{2}$ CAS Key Laboratory of Nano-Bio Interface, Suzhou Institute of Nano-Tech and Nano-Bionics, Chinese Academy \\ of Sciences, Suzhou, Jiangsu, China; ${ }^{3}$ Livingchip Lnc., Nanjing, Jiangsu, China; ${ }^{4}$ Center for Clinic Stem Cell Research, \\ The Affiliated Drum Tower Hospital of Nanjing University Medical School, Nanjing, Jiangsu, China
}

\begin{abstract}
Mesenchymal stem cells (MSCs) have shown great potentials in regenerative medicine for their low immunogenicity, multilineage differentiation potential, and extensive sources. However, the heterogeneity of MSCs limits their clinical application and industrial prospects. In this review, we introduced the heterogeneity of MSCs in terms of their applications, sources, functions, and surface markers; discussed the major factors leading to the heterogeneity in MSCs; summarized the main approaches to study the MSC heterogeneity, and addressed the clinical challenges resulting from heterogeneity. Finally, we proposed the strategies that might be used to purify the MSCs and to eliminate the heterogeneity of MSCs for their standardized production and reliable clinical application.
\end{abstract}

Keywords: heterogeneity; mesenchymal stem cell; regenerative medicine; cell therapy

Received: 8 October 2021; Revised: 8 November 2021; Accepted: I I November 2021; Published: 05 January 2022

$\mathrm{M}$ esenchymal stem cells (MSCs) are one kind of trophoblast cells that exists in most adult connective tissues. MSCs contribute to the maintenance and regeneration of diverse tissue by differentiating into bone, fat, cartilage, fibers and so on. These cells can secrete cytokines to support the survival and growth of various cells (1).

Mesenchymal stem cells are isolated from various tissues, such as amniotic membrane, dental pulp, fat, umbilical cord blood, umbilical cord, thymus, peripheral blood, and bone marrow (Table 1). The MSCs are defined by the International Society of Cellular Therapy as a heterogeneous population of spindle-shaped cells having minimum characteristics: (1) adherent growth; (2) expression of CD73, CD1053, and CD 90 ( $295 \%$, flow cytometry detection) and negative expression of CD14, HLA-DR, CD34, CD11b or CD45, CD19 or CD $79 \alpha(\leq 2 \%$, flow cytometry detection); and (3) differentiation into chondrocytes, osteoblasts, and adipocytes (2).
Numerous studies proved that MSCs could maintain stem cell properties even after multiple passages and have anti-bacterial, anti-fibrosis, and anti-transplant rejection properties (3-5). MSCs continuously secrete anti-apoptotic and anti-inflammatory cytokines to construct a favorable microenvironment that promotes tissue repair and regeneration in vivo (6). These cells are easily harvested, separated, cultured, and amplified in vitro. Benefiting from these advantages, MSCs are hopefully applied for tissue engineering and cell reprogramming. MSCs have been the most widely used adult stem cells in clinic. So far, there have been over 1000 registered clinical trials regarding MSCs around the world (www.clinicaltrials.gov), including pulmonary fibrosis (7), bone tissue engineering $(8,9)$, cartilage tissue engineering (10-12), myocardial infarction (MI) $(13,14)$, systemic lupus erythematosus (15), graft-versus-host disease (GVHD) (16), spinal cord injury $(17,18)$, hematopoiesis (19-21), diabetic foot ulcer (22), and multiple sclerosis (23). It is noteworthy that for their

tThese authors are equal contribution to this work 
immune-modulatory properties, MSCs have been used for clinical therapy of coronavirus disease 2019 (COVID-19), a contagious disease induced by severe acute respiratory syndrome coronavirus 2 (SARS-CoV-2) (24-30). Preliminary clinical data indicated that the MSCs alleviated the clinical symptoms of COVID-19 patients by reducing cytokine storms, increasing oxygen saturation, and regenerating lung tissue $(25,31-36)$.

For the great potential of clinical application, the market of MSCs is now booming. According to the data released by Research and Markets, the global market of MSCs is approximately $\$ 172.9$ million in 2020 and is expected to grow at a compound annual growth rate of $4.1 \%$ in the next 7 years. The market is expected to reach a revised size of \$229 million by 2027. In February 2018, the company of Mesoblast Limited announced that the Phase 3 trial of the allogeneic MSCs for treating children with steroid-refractory acute graft-versus-host disease (aGVHD) achieved success. Although people are optimistic about the commercial prospects of MSCs, so far, most applications of MSCs have been in clinical trials (37). In comparison with the great advantages of MSCs for cell therapy, the clinical application and industrial development of MSCs are still far from satisfactory. One of the main impeding factors is heterogeneity. Usually, cell-based therapeutic products require stable biological characteristics to obtain long-lasting therapeutic effects among patients undergoing treatment. So, the safety and curative effect of MSCs have always been the most critical criterion in clinical applications. Due to MSC heterogeneity, it is difficult to establish a criterion for patient selection and provide repeatable treatment plans for patients. In manufacture, the heterogeneity impedes the large-scale standardized production of MSCs with high and uniform quality.

In this review, we discuss the major factors leading to the heterogeneity in MSCs, summarize the main approaches to study the MSC heterogeneity, and address the clinical challenges resulting from heterogeneity. Finally, we propose the strategies that may be used to purify MSCs and to eliminate the heterogeneity of MSCs for their standardized production and reliable clinical application.

\section{Heterogeneity of MSCs}

Mesenchymal stem cell heterogeneity means the differences in cell morphology and function among the heterogeneous subpopulation. This heterogeneity is inherent among donors (sex and age, etc.), source tissues (umbilical cord, gums, placenta, bone marrow, dental pulp, and adipose tissue, etc.), isolation and culture methods, passage times, and treatment modality (cryopreserved, resuscitation, etc.). These factors contribute to the primary cultured MSCs' high heterogeneity of proliferation potential, secretion of factors, and immune regulation capacity (Fig. 1) $(38,39)$.

It has been proved that there are differences in the immunoregulatory function of MSCs from different tissues and individuals (40). For example, MSCs derived from high-risk myelodysplastic syndrome were associated with higher immunosuppressive and apoptosis rates compared with MSCs derived from low-risk myelodysplastic syndrome (41). The individual heterogeneity of MSCs indicates their different therapeutic effects on different diseases. Therefore, many efforts were made to uncover the heterogeneity of MSCs.

\section{Heterogeneity among individual donors}

Numerous studies have revealed that MSCs are heterogeneous among different individuals. MSCs derived from the same tissue from different individuals also have heterogeneity. Xie et al. analyzed the features of hUC-MSCs derived from multiple donors, including cell activity, surface marker, multi-differentiation potential, immunoregulatory capability, and so on. Next, they treated mouse liver fibrosis using the hUC-MSCs with distinct immunomodulatory effects. The results showed that hUC-MSCs from multiple donors displayed a substantial heterogeneity in multi-differentiation potential and immunoregulatory capability; however, they have similar surface marker expression patterns, survival rate, growth curve, and tumorigenicity. In vivo results showed that three hUC-MSCs alleviated liver fibrosis; however, three hUC-MSCs have different therapeutic effects. The repair effects were dependent on the immune regulation ability of MSCs (40).

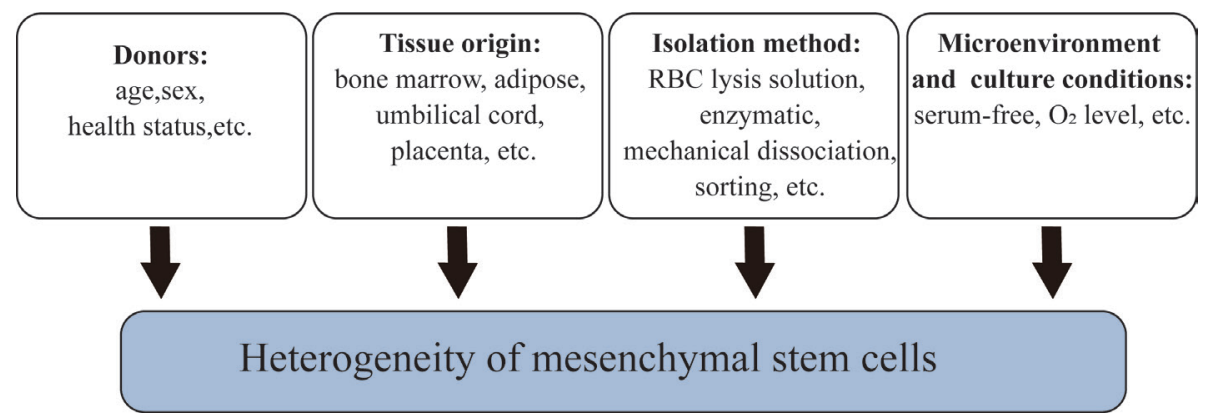

Fig. 1. Heterogeneity of mesenchymal stem cells. 
It was reported that there are significant differences in the growth rate, alkaline phosphatase activity, and bone-specific gene level of bone marrow MSCs (BMMSCs) from 17 healthy donors (42). MSCs in the bone marrow of inbred strains of mice exhibit markedly disparate expression levels of alkaline phosphatase that is an early marker of osteoblast differentiation (43). The age of the donors also affects the heterogeneity of MSCs (44). Zhou et al. found that in the older subjects ( $>55$ years), the proliferation and osteoblast differentiation of MSCs were significantly decreased. The senescence-associated $\beta$-galactosidase (SA- $\beta$-gal) activity and the expression of p53 pathway genes in older subjects were higher than in young subjects. The proportion of SA- $\beta$-gal ${ }^{+}$MSCs derived from older subjects were four times higher than that of younger subjects. The proliferation rate of MSCs derived from the older subjects is 1.7 times less than that of the younger subjects (44). The human umbilical cord MSCs (hUC-MSCs) from different people have different sensitivity to hypoxic conditions. The result of bioinformatics analysis demonstrated the intrinsic variability and suggested that the alternative potential genetic factors, such as ADM, ANGPTL4, CDON, and SLC2A3, may be considered as potential indicators for cell therapy (45). Genders can affect the heterogeneity of MSCs. Xie et al. revealed that the osteogenic potential of male infant hUC-MSCs was approximately 10 times higher than that of female infant hUC-MSCs in vitro. On the contrary, there was no apparent heterogeneity in cartilage and adipogenesis differentiation capacity (40).

\section{Heterogeneity of MSCs under different culturing environments}

A critical factor determining the heterogeneity of MSCs may be the microenvironment of MSCs (46). MSC functions are extremely diverse and depend on the special microenvironment in which MSCs are embedded (47). A hypoxic environment would induce hypoxia inducible factor-1a (HIF-1a) expression of MSCs. In a $1 \%$ hypoxic environment, MSCs tend to be elliptical and up-regulate the expression of stemness-associated markers, such as octamer-binding transcription factor 4 (OCT4), NANOG, and SRY-related high-mobility-group-box protein 2 (SOX2) (48). Studies had shown that the growth rate of MSCs and stemness were significantly enhanced when adipose-derived MSCs (AD-MSCs) were cultured under $1 \%$ hypoxic conditions (49). However, hypoxic culture could inhibit the osteogenic differentiation and adipogenic differentiation of MSCs. When the oxygen content was increased from 1 to $3 \%$, the osteogenic differentiation ability of MSCs was restored (50). The serum may affect the heterogeneity of MSCs. There were differences in the cell proliferation, telomerase, and gene expression profile when hUC-MSCs were cultured in serum or serum-free medium (SFM). In the SFM, the hUC-MSCs had a slower proliferation rate, increased cell apoptosis, and higher pluripotency. Both SFM- and serum-containing medium-expanded hUC-MSCs finally obtained copy number variant in the long-term in vitro culture (51). In addition, adding a small amount of tumor necrosis factor- $\alpha$ (TNF- $\alpha)$, interleukin-6 (IL-6), and interferon- $\gamma$ (IFN- $\gamma$ ) to the culture medium enhanced the immunosuppressive function of AD-MSCs $(52,53)$. Otherwise, studies reported that the mechanical forces from extracellular matrix (ECM) materials and three-dimensional (3D) culture environment would affect cell polarity and cell-cell interactions, and further affect biochemical signaling, gene expression, and cell phenotype, finally contributing to the heterogeneity of $\operatorname{MSCs}(54,55)$.

\section{Heterogeneity among different tissue origins}

Mesenchymal stem cells derived from various tissues will exhibit different cytological characteristics. So far, many sources of MSCs have been applied for cellular therapy (Table 1).

Araújo et al. interrogated the biological characteristics of different MSCs, such as AM-MSCs, PD-MSCs, CMMSCs, and UC-MSCs, and analyzed their differentiation ability, immunophenotype, cell complexity, cell size,

Table 1. The source of MSCs in the body

\begin{tabular}{ll}
\hline Source & \\
\hline I. Bone marrow & (BM-MSC) \\
2. Placenta & (PD-MSC) \\
3. Peripheral blood & (PB-MSC) \\
4. Wharton's Jelly & (WJ-MSC) \\
5. Adipose tissue & (AD-MSC) \\
6. Fetal pancreas & (FPan-MSC) \\
7. Chorionic plate & (CP-MSC) \\
8. Breast milk & (Br-MSC) \\
9. Chorionic villi & (CP-MSC) \\
10. Placental villi & (PV-MSC) \\
II. Dermal sheath cell & (DSC) \\
12. Placental Decidua basalis & (PDB-MSC) \\
13. Amniotic membrane & (AM-MSC) \\
14. Ligamentum flavum & (LF-MSC) \\
I5. Amniotic fluid & (AF-MSC) \\
16. Dental pulp & (DP-MSC) \\
17. Umbilical cord & (UC-MSC) \\
18. Menstrual blood & (Men-MSC) \\
19. Umbilical cord blood & (UCB-MSC) \\
20. Arthroscopic flushing fluid & (AFF-MSC) \\
21. Umbilical cord lining & (UCL-MSC) \\
22. Dermal papilla & (DPC) \\
23. Umbilical cord matrix & (UCM-MSC) \\
\hline
\end{tabular}


polarity index, and growth kinetics (56). Although the surface marker profile and differentiation ability of all sources of MSCs were consistent with the characteristics of human MSCs, they had far differences in cell size, morphology, polarity, growth capacity, proliferation, and lifespan. The other research study reported that there were significant differences in phenotype, proliferation, migration, and immunogen among Wharton's jelly MSCs (WJ-MSCs), UCB-MSCs, PD-MSCs, and human umbilical cord lining MSCs (UCL-MSCs). UCL-MSCs showed the highest proliferation and migration rate but a lower cellular immune response. UCB-MSCs and WJ-MSCs significantly enhanced the release of IFN- $\gamma$ (57). Kern et al. revealed that BM-MSCs had the shortest proliferation capacity, followed by AD-MSCs. However, UCB-MSCs showed the proliferation capacity in all passages analyzed (58).

The expression patterns of paracrine factors are also different among the different sources of MSCs. One of the research studies reported that although the secretion levels of angiogenin and vascular endothelial growth factor-A (VEGF-A) in MSC populations isolated from AD-MSCs, BM-MSCs, dermal sheath cells (DSCs), and dermal papillary cells (DPCs) were almost similar, the expression levels of insulin-like growth factor-1 (IGF-1), VEGF-D, and IL-8 in AD-MSCs were higher than other MSCs, suggesting their different therapeutic potential in clinic (59).

Otherwise, studies reported that MSCs derived from placental villi (PV-MSCs) had better immunomodulatory and pro-angiogenic activities than BM-MSCs, AD-MSCs, and UC-MSCs (60); UC-MSCs had a higher proportion of neuron-specific enolase-positive cells than BM-MSCs when they were induced to neurons (61). Human umbilical cord perivascular cells (hUCPVCs) had higher pluripotency and the expression of CD146 than BM-MSCs and could be more easily differentiated into osteogenic, cartilage, and adipogenesis (62); UC-MSCs promoted megakaryocyte production, while BM-MSCs could expand more megakaryocyte progenitor cells from $\mathrm{CD} 34^{+}$ hematopoietic precursor cells (63).

\section{Heterogeneity of MSCs caused by different isolation methods}

Different techniques have been used for MSC isolation. These techniques can affect the quantity and quality of the MSCs, and contribute to MSC heterogeneity (64). There are two main methods to obtain MSCs, including the enzymatic digestion and explant method. Hua et al. found that the proliferation rate of MSCs in the explant cure treated was higher than the MSCs in the enzymatic group. They found that the MSCs in the $10-\mathrm{mm}$ size pieces group (explant cure methods) exhibited the highest number of cells, shortest primary culture time, and highest proliferation rates (65).
In another study, Horn et al. compared the isolation of BM-MSCs based on red blood cell (RBC) lysis with ammonium chloride, Ficoll density fractionation, and colony formation from untreated whole bone marrow. The colonies were larger through the RBC lysis method than through Ficoll density gradient separation, which might arise from the platelets maintained after the RBC lysis isolation. RBC lysis can be standardized more efficiently and faster than the Ficoll procedure for the clinical application of MSCs (66). The MSCs isolated with human platelet lysate (HPF) showed a higher osteogenic capability and proliferation ability than those treated with the fetal calf serum (67).

\section{Markers involved in MSCs heterogeneity}

Mesenchymal stem cells derived from bone marrow, fat, umbilical cord, and umbilical blood have significant differences in the expression of cell surface markers, such as CD271, Stro-1 (68), CD146, CD106 (69), nestin (70), GD2, and SSEA-4 $(71,72)$. Currently, these MSC surface markers may offer a breakthrough to reveal the heterogeneity of MSCs.

Stro-1 is one of well-known MSCs markers; however, it is not universally expressed in all reported types of MSCs (73). Immature MSCs have high proliferative rates and multi-differentiation potential and are associated with the high expression of Stro-1. The researcher showed that Stro- $1^{+}$cells tend to have higher homing capabilities and gene delivery functions, while Stro- $1^{-}$cells support hematopoietic engraftment to a greater extent (74).

CD271 is highly expressed in BM- or AD-MSCs, not expressed in UC-MSCs and lowly expressed in placenta-derived MSCs (PD-MSCs). Studies have shown that $\mathrm{CD} 271^{+}$hematopoietic stem cells (HSCs) and CD133 ${ }^{+}$ HSCs are co-transplanted into NOD/SCID-IL2R $\gamma$ (null) mice at a ratio of $8: 1, \mathrm{CD} 271^{+}$HSCs have significantly promoting effects on lymphocyte engraftment compared with $\mathrm{CD} 133^{+}$HSCs (75).

CD146 ${ }^{+}$MSCs represented an MSC subpopulation that support hematopoiesis and secrete growth factors controlling the function of HSCs. CD146 PDGF-R $\beta^{+}$ MSCs possessed a stronger self-renewal ability and can differentiate into adipocytes and osteoblasts compared with CD146-PDGF-R $\beta$ - cells (76).

CD106 is an adhesion protein, which is essential for the immunosuppression mediated by BM-MSCs and the binding of HPCs. Combining THY-1, LNGFR and CD106 can efficiently select BM-MSCs (77). Compared with the $\mathrm{CD} 106^{-}$cells, the $\mathrm{CD} 106^{+}$cells contained fewer osteoblasts and more adipocytes, indicating that CD106 can be used as a predictive indicator for the differentiation of BM-MSCs (78).

SSEA-4, an embryonic stem cell marker, is a marker of BM-MSCs and is used to isolate genuine MSCs from 
bone marrow (79). On the contrary, the AT-MSCs, UCMSCs, and UB-MSCs do not express SSEA-4 $(80,81)$. SSEA-4- BMSCs failed to grow, while SSEA-4+ ${ }^{+}$BMSCs expand extensively. Moreover, the expression of SSEA-4 in BMSCs gradually increased over time (79).

\section{The pros and cons of heterogeneity in the clinical application of MSCs}

So far, MSC-based therapy has been widely used in clinic. However, how the heterogeneity affects the clinical application of MSCs is still controversial (82). In 2009, the use of an industrial MSC product failed to meet its primary clinical end point of achieving a significant increase of complete response of SR-aGvHD (NCT00366145) (83). The failure may result from the limitation of MSCs diversity. All MSCs used in the phase-3 trial were derived from a single donor, and the MSCs were expanded to passages 3 and 4 to yield enough MSCs to treat all 240 participants. On the contrary, in the other research, Kuçi et al. treated $26 \mathrm{SR}-\mathrm{aGvHD}$ patients with pooled MSCs generated from multiple healthy donors. Finally, they got the result that a 77\% overall response in GvHD patients was induced at the primary end point, which was much better than the result treated with MSCs derived from single donors (84). Although the pooled MSCs seem to circumvent the donor-to-donor heterogeneity, it is not as what the authors stated that this method can minimize the heterogeneity. In fact, this method certainly magnified the heterogeneity rather than solving the problem. The pooled method may be just expediency for therapy before clearly understanding the heterogeneity and purifying the MSCs.

For a long time, scientists have been dying to know whether different sources of MSCs are specifically beneficial to different indications. Thus, the study on the heterogeneity of MSCs derived from different tissue sources is quite significant to answer these questions.

\section{Current methods used for the study of MSCs heterogeneity}

The single-cell RNA-sequencing technology was regarded as one of the most powerful tools to investigate cell heterogeneity under various conditions (85). Hou et al. found that MSCs derived from the four tissues (BM, UC, adipose tissue, and synovial tissue) possessed different trilineage differentiation potentials through single-cell RNA-sequencing analysis (86). They found that UC-MSCs exhibited the highest immunosuppression. The differentiation potentials of MSC subsets showed significant differences in each other, and are strongly associated with their subtypes and tissue sources. Huang et al. analyzed the 361 single-cell transcriptomes of MSCs derived from two samples harvested at different passages and stimulated with or without inflammatory cytokines. The single-cell RNA-sequencing revealed the existence of subsets in hUC-MSCs, and these subsets were conserved independent of donors and passages. In addition, the expression of key cytokines and chemokines for MSCs-mediated immune modulation showed a similar expression pattern at different passages ( $00, \mathrm{p} 2, \mathrm{p} 5)$ at the persistence of inflammatory factors (87).

Efforts have been made to explore the characteristics and differences of AD-MSCs and BM-MSCs at the singleand bulk-cell assays and further study the clinical effects of two types of cells in treating osteoarthritis. Single-cell RNA sequencing of AD-MSCs and BM-MSCs showed that the transcriptional heterogeneity of AD-MSCs cell population is lower than that of BM-MSCs. Furthermore, compared with BM-MSCs, AD-MSCs were less dependent on mitochondrial respiratory energy supply. Furthermore, AD-MSCs had a lower HLA I antigen expression level and higher immunosuppressive ability. Meta-analysis of current clinical trials using BMSCs to treat osteoarthritis showed that the therapeutic effect of ADMSCs is more stable than that of BM-MSCs. AD-MSCs may be a more controllable source of stem cells, more suitable for survival in hypoxic joint cavities, and may be more advantageous in regulating inflammation (88).

Besides the single-cell sequencing, other technologies based on single-cell or colony assays were generated for the research field. It was reported that a single-cell FTIR microspectroscopy-based method was developed for the quantitative analysis of cellular heterogeneity by quantitatively calculating the cell-to-cell Euclidean distance. The result showed that the size, shape, and chemical component spatial distribution patterns exhibited remarkable heterogeneity among the different cell populations (89). Russell et al. developed a high-capacity assay to quantify the clonal heterogeneity of MSCs. The MSCs were classified according to colony-forming efficiency and quantified the trilineage differentiation potentials of MSC clones. The authors reported that the heterogeneity in the trilineage potential of normal BM-MSCs is more complex than previously reported (90). Rennerfeldt et al. quantified the heterogeneity that emerged over multiple cell divisions by observing BM-MSC colony formation in real time using time-lapsed optical imaging and analysis. They found that the cells in the subpopulation are pure initially but became functionally heterogeneous after cultured (91).

Proteomic methods have been extensively used to analyze MSC heterogeneity on the expression of proteins on cell surface that may be changed due to the passages (92). For example, FACSCAP Lyoplate proteomic analysis system has been used to research the expression changes of cell surface proteins in BM-MSCs through different culture passages (93).

Furthermore, besides the methods above mentioned, microfluidic technologies were used for the study of MSC heterogeneity $(94,95)$. It is well known that MSC 
morphology and size may indicate differentiation potential of MSCs. Liu et al. developed a high-throughput microfluidic platform to analyze the MSC heterogeneity according to the cell size (96). They found that larger MSCs with slow proliferation displayed a senescent phenotype.

\section{The new efforts for resolving the heterogeneity of MSCs}

Mesenchymal stem cell heterogeneity is one of the greatest challenges for cell therapy and regenerative medicine. A lack of technologies or methods to dissect and resolve heterogeneity of MSCs greatly impeded the application of MSCs in the clinic and their standardized production. Although many efforts have been made to study the heterogeneity of MSCs, most works just focused on discovering the existence of heterogeneity and understanding how heterogeneous the MSCs are. However, many attempts have been made to establish the evaluation standard through bioinformatics analysis by mining the mass data of cell sequencing. However, these works were largely confined to existing biological knowledge. Controlling or eliminating the inherent heterogeneity of MSCs is the ultimate aim of this research field, is a crucial requirement to achieve the curative effect of MSC-based therapies, and is a premise of the industrialization of MSCs. Recently, new efforts have been made in this respect.

The idea of induced pluripotent stem cell (iPSC) generation have been used to produce highly uniform MSCs. The iPSCs may have the potential to overcome heterogeneity due to their capacity for infinite proliferation and multilineage differentiation. Recently, a kind of iPSC-derived MSCs from a single donor was produced by Ctmerus $^{\mathrm{TM}}$ for the therapy of steroid-resistant acute graft versus host disease (SR-aGvHD) (97). The iPSC-MSC potency is consistent with MSCs of earlier generations undergoing the $\mathrm{CD}^{+}$T-cell potency assay (98). Although iPSC-derived MSCs took a big step forward for the manufacturing of MSCs, they still cannot be a substitute for the genuine MSCs. Moreover, transgene technologies used for the generation of iPSC-derived MSCs caused new problems such as off-target effects and high cytotoxicity (99-101).

In our recent study, a novel approach was developed to produce single-cell-derived sphere (SCDS) that can reflect the potential of stem cells in vitro (102). SCDSs can be formed due to the self-renewal ability of single cells. To high throughput produce hUC-MSC SCDSs, special cell chips were fabricated for single hUC-MSCs two-dimensional (2D) patterning and 3D culturing for several days. Some hUC-MSCs formed SCDSs for their higher self-renewal ability. Compared with 2D cultured hUC-MSCs, 3D SCDSs had an enhanced self-renewal ability, multi-directional differentiation potential, anti-aging, anti-stress, migration ability, survival ability, and paracrine effects in vitro. The SCDS cultured hUC-MSCs remarkably promoted angiogenesis in vivo and displayed greater therapeutic potential on acute liver failure (ALF) in mice. This method based on cell chip screening provided a tool to purify the hUC-MSCs according to the self-renewal ability and also may offer a possibility to eliminate the inherent heterogeneity of MSCs.

\section{Perspective}

Although many preclinical and clinical studies have demonstrated the great potential of MSCs for cell therapy of various diseases, inherent heterogeneity is the main obstacle to impede MSCs' clinical application and industrialization. High-throughput sequencing (HTS), especially single-cell based HTS, offers a powerful tool to discover and evaluate the heterogeneity of MSCs. However, controlling and eliminating heterogeneity are the ultimate goals of the research field. In future, more technologies regarding the functional or phenotypic purification of MSCs need to be developed. Through purification, the functional or phenotypic heterogeneity of MSCs may be efficiently eliminated, and the quintessential and high-quality MSCs with higher self-renewal, differentiation potential, and paracrine abilities may be screened out from the bulk of MSCs. Integrating single-cell-based HTS, cell function assays, animal tissue repair experiments and clinical evaluation, the quality assessment standard of MSCs and indication selection criteria of cell therapy for different MSCs can be well established. Relying on these criteria, the standardized production of high-quality MSCs can be carried out in industrialization, and different sources of MSCs can be classified to meet different indications in clinic.

\section{Acknowledgments}

The authors thank the technical service provided by Livingchip Inc.

\section{Conflict of interest and funding}

The authors declare that there are no conflicts of interest. This research work was supported by funds from Ministry of Science and Technology (MOST) of China (Grant No. 2017YFA0104301); National Natural Science Foundation of China (Grant No. 31870975), Natural Science Foundation of Jiangxi Province (Grant No. 20192ACBL20053), Key Project of Jiangsu Province (Grant No. BE2020765), Strategic Priority Research Program of the Chinese Academy of Sciences (Grant No. XDA16020103), and Natural Science Foundation of Jiangsu Province (Grant No. SBK2021040505).

\section{References}

1. Chamberlain G, Fox J, Ashton B, Middleton J. Concise review: mesenchymal stem cells: their phenotype, differentiation capacity, immunological features, and potential for homing. Stem Cells 2007; 25(11): 2739-49. doi: 10.1634/stemcells.2007-0197 
2. Dominici M, Le Blanc K, Mueller I, Slaper-Cortenbach I, Marini F, Krause D, et al. Minimal criteria for defining multipotent mesenchymal stromal cells. The International Society for Cellular Therapy position statement. Cytotherapy 2006; 8(4): 315-17. doi: 10.1080/14653240600855905

3. Alcayaga-Miranda F, Cuenca J, Khoury M. Antimicrobial activity of mesenchymal stem cells: current status and new perspectives of antimicrobial peptide-based therapies. Front Immunol. 2017; 8: 339. doi: 10.3389/fimmu.2017.00339

4. Cselenyák A, Pankotai E, Horváth EM, Kiss L, Lacza Z. Mesenchymal stem cells rescue cardiomyoblasts from cell death in an in vitro ischemia model via direct cell-to-cell connections. BMC Cell Biol 2010; 11: 29. doi: 10.1186/1471-2121-11-29

5. Zhang LB, He M. Effect of mesenchymal stromal (stem) cell (MSC) transplantation in asthmatic animal models: a systematic review and meta-analysis. Pulm Pharmacol Ther 2019; 54: 39-52. doi: 10.1016/j.pupt.2018.11.007

6. Caplan AI, Correa D. The MSC: an injury drugstore. Cell Stem Cell 2011; 9(1): 11-15. doi: 10.1016/j.stem.2011.06.008

7. Li F, Han F, Li H, Zhang J, Qiao X, Shi J, et al. Human placental mesenchymal stem cells of fetal origins-alleviated inflammation and fibrosis by attenuating MyD88 signaling in bleomycin-induced pulmonary fibrosis mice. Mol Immunol. 2017; 90: 11-21. doi: 10.1016/j.molimm.2017.06.032

8. Ismail HD, Phedy P, Kholinne E, Djaja YP, Kusnadi Y, Merlina $\mathrm{M}$, et al. Mesenchymal stem cell implantation in atrophic nonunion of the long bones: a translational study. Bone Joint Res 2016; 5(7): 287-293. doi: 10.1302/2046-3758.57.2000587

9. Suenaga H, Furukawa KS, Suzuki Y, Takato T, Ushida T. Bone regeneration in calvarial defects in a rat model by implantation of human bone marrow-derived mesenchymal stromal cell spheroids. J Mater Sci Mater Med 2015; 26(11): 254. doi: 10.1302/2046-3758.57.2000587

10. Zhang S, Chuah SJ, Lai RC, Hui JHP, Lim SK, Toh WS. MSC exosomes mediate cartilage repair by enhancing proliferation, attenuating apoptosis and modulating immune reactivity. Biomaterials 2018; 156: 16-27. doi: 10.1016/j. biomaterials.2017.11.028

11. Zhang K, Yan S, Li G, Cui L, Yin J. In-situ birth of MSCs multicellular spheroids in poly(L-glutamic acid)/chitosan scaffold for hyaline-like cartilage regeneration. Biomaterials 2015; 71: 24-34. doi: 10.1016/j.biomaterials.2015.08.037

12. Li J, Huang Y, Song J, Li X, Zhang X, Zhou Z, et al. Cartilage regeneration using arthroscopic flushing fluid-derived mesenchymal stem cells encapsulated in a one-step rapid cross-linked hydrogel. Acta Biomater 2018; 79: 202-15. doi: 10.1016/j. biomaterials.2015.08.037

13. Huang $\mathrm{P}$, Wang $\mathrm{L}$, Li Q, Xu J, Xu J, Xiong $\mathrm{Y}$, et al. Combinatorial treatment of acute myocardial infarction using stem cells and their derived exosomes resulted in improved heart performance. Stem Cell Res Ther 2019; 10(1): 300. doi: 10.1186/ s13287-019-1353-3

14. Park TY, Oh JM, Cho JS, Sim SB, Lee J, Cha HJ. Stem cellloaded adhesive immiscible liquid for regeneration of myocardial infarction. J Control Release 2020; 321: 602-15. doi: 10.1186/s13287-019-1353-3

15. Zhou T, Li HY, Liao C, Lin W, Lin S. Clinical efficacy and safety of mesenchymal stem cells for systemic lupus erythematosus. Stem Cells Int 2020; 2020: 6518508. doi: 10.1155/2020/6518508

16. Zhao L, Chen S, Yang P, Cao H, Li L. The role of mesenchymal stem cells in hematopoietic stem cell transplantation: prevention and treatment of graft-versus-host disease. Stem Cell Res Ther 2019; 10(1): 182. doi: 10.1155/2020/6518508
17. Peng Z, Gao W, Yue B, Jiang J, Gu Y, Dai J, et al. Promotion of neurological recovery in rat spinal cord injury by mesenchymal stem cells loaded on nerve-guided collagen scaffold through increasing alternatively activated macrophage polarization. J Tissue Eng Regen Med 2018; 12(3): e1725-36. doi: $10.1155 / 2020 / 6518508$

18. Wu GH, Shi HJ, Che MT, Huang MY, Wei QS, Feng B, et al. Recovery of paralyzed limb motor function in canine with complete spinal cord injury following implantation of MSC-derived neural network tissue. Biomaterials 2018; 181: 15-34. doi: 10.1016/j.biomaterials.2018.07.010

19. Almeida-Porada G, Porada CD, Tran N, Zanjani ED. Cotransplantation of human stromal cell progenitors into preimmune fetal sheep results in early appearance of human donor cells in circulation and boosts cell levels in bone marrow at later time points after transplantation. Blood 2000; 95(11): 3620-7. doi: 10.1182/blood.V95.11.3620

20. Noort WA, Kruisselbrink AB, in't Anker PS, Kruger M, Van Bezooijen RL, De Paus RA, et al. Mesenchymal stem cells promote engraftment of human umbilical cord blood-derived CD34(+) cells in NOD/SCID mice. Exp Hematol 2002; 30(8): 870-8. doi: 10.1016/S0301-472X(02)00820-2

21. Koç ON, Gerson SL, Cooper BW, Dyhouse SM, Haynesworth SE, Caplan AI, et al. Rapid hematopoietic recovery after coinfusion of autologous-blood stem cells and culture-expanded marrow mesenchymal stem cells in advanced breast cancer patients receiving high-dose chemotherapy. J Clin Oncol 2000; 18(2): 307-16. doi: 10.1200/JCO.2000.18.2.307

22. Shi R, Lian W, Jin Y, Cao C, Han S, Yang X, et al. Role and effect of vein-transplanted human umbilical cord mesenchymal stem cells in the repair of diabetic foot ulcers in rats. Acta Biochim Biophys Sin (Shanghai) 2020; 52(6): 620-30. doi: 10.1093/abbs/gmaa039

23. Petrou P, Kassis I, Levin N, Paul F, Backner Y, Benoliel T, et al. Beneficial effects of autologous mesenchymal stem cell transplantation in active progressive multiple sclerosis. Brain 2020; 143(12): 3574-88. doi: 10.1093/brain/awaa333

24. Liang B, Chen J, Li T, Wu H, Yang W, Li Y, et al. Clinical remission of a critically ill COVID-19 patient treated by human umbilical cord mesenchymal stem cells: a case report. Medicine (Baltimore) 2020; 99(31): e21429. doi: 10.1097/ MD.0000000000021429

25. Leng Z, Zhu R, Hou W, Feng Y, Yang Y, Han Q, et al. Transplantation of ACE2(-) mesenchymal stem cells improves the outcome of patients with COVID-19 pneumonia. Aging Dis 2020; 11(2): 216-28. doi: 10.1097/MD.0000000000021429

26. Atluri S, Manchikanti L, Hirsch JA. Expanded umbilical cord mesenchymal stem cells (UC-MSCs) as a therapeutic strategy in managing critically Ill COVID-19 patients: the case for compassionate use. Pain Physician. 2020; 23(2): E71-83. doi: 10.36076/ ppj.2020/23/E71

27. Liu S, Peng D, Qiu H, Yang K, Fu Z, Zou L. Mesenchymal stem cells as a potential therapy for COVID-19. Stem Cell Res Ther 2020; 11(1): 169. doi: 10.1186/s13287-020-01678-8

28. Coelho A, Alvites RD, Branquinho MV, Guerreiro SG, Maurício AC. Mesenchymal stem cells (MSCs) as a potential therapeutic strategy in COVID-19 patients: literature research. Front Cell Dev Biol 2020; 8: 602647. doi: 10.3389/fcell.2020.602647

29. Rogers CJ, Harman RJ, Bunnell BA, Schreiber MA, Xiang C, Wang FS, et al. Rationale for the clinical use of adipose-derived mesenchymal stem cells for COVID-19 patients. J Transl Med 2020; 18(1): 203. doi: 10.3389/fcell.2020.602647

30. Zumla A, Wang FS, Ippolito G, Petrosillo N, Agrati C, Azhar EI, et al. Reducing mortality and morbidity in patients with severe 
COVID-19 disease by advancing ongoing trials of Mesenchymal Stromal (stem) Cell (MSC) therapy - achieving global consensus and visibility for cellular host-directed therapies. Int J Infect Dis 2020; 96: 431-9. doi: 10.1016/j.ijid.2020.05.040

31. Kaffash Farkhad N, Reihani H, Sedaghat A, Moghadam AA, Moghadam AB, Tavakol-Afshari J. Are mesenchymal stem cells able to manage cytokine storm in COVID-19 patients? A review of recent studies. Regen Ther 2021; 18: 152-60. doi: 10.1016/j. ijid.2020.05.040

32. Shu L, Niu C, Li R, Huang T, Wang Y, Huang M, et al. Treatment of severe COVID-19 with human umbilical cord mesenchymal stem cells. Stem Cell Res Ther 2020; 11(1): 361. doi: 10.1016/j.ijid.2020.05.040

33. Shi L, Huang H, Lu X, Yan X, Jiang X, Xu R, et al. Effect of human umbilical cord-derived mesenchymal stem cells on lung damage in severe COVID-19 patients: a randomized, double-blind, placebo-controlled phase 2 trial. Signal Transduct Target Ther. 2021; 6(1): 58. doi: 10.1016/j.ijid.2020.05.040

34. Kouroupis D, Lanzoni G, Linetsky E, Messinger Cayetano S, Wishnek Metalonis S, Leñero C, et al. Umbilical cord-derived mesenchymal stem cells modulate TNF and soluble TNF receptor 2 (sTNFR2) in COVID-19 ARDS patients. Eur Rev Med Pharmacol Sci 2021; 25(12): 4435-8. doi: 10.26355/eurrev_202106_26156

35. Meng F, Xu R, Wang S, Xu Z, Zhang C, Li Y, et al. Human umbilical cord-derived mesenchymal stem cell therapy in patients with COVID-19: a phase 1 clinical trial. Signal Transduct Target Ther 2020; 5(1): 172. doi: 10.1016/j.ijid.2020.05.040

36. Chen X, Shan Y, Wen Y, Sun J, Du H. Mesenchymal stem cell therapy in severe COVID-19: a retrospective study of short-term treatment efficacy and side effects. J Infect 2020; 81(4): 647-79. doi: 10.1016/j.jinf.2020.05.020

37. Galipeau J, Sensébé L. Mesenchymal stromal cells: clinical challenges and therapeutic opportunities. Cell Stem Cell 2018; 22(6): 824-33. doi: 10.1016/j.stem.2018.05.004

38. Le Blanc K, Ringdén O. Immunomodulation by mesenchymal stem cells and clinical experience. J Intern Med 2007; 262(5): 509-25. doi: 10.1111/j.1365-2796.2007.01844.x

39. Levy O, Kuai R, Siren EMJ, Bhere D, Milton Y, Nissar N, et al. Shattering barriers toward clinically meaningful MSC therapies. Sci Adv 2020; 6(30): eaba6884. doi: 10.1126/sciadv.aba6884

40. Xie Y, Liu S, Wang L, Yang H, Tai C, Ling L, et al. Individual heterogeneity screened umbilical cord-derived mesenchymal stromal cells with high Treg promotion demonstrate improved recovery of mouse liver fibrosis. Stem Cell Res Ther 2021; 12(1): 359. doi: 10.1186/s13287-021-02430-6

41. Zhao Z, Wang Z, Li Q, Li W, You Y, Zou P. The different immunoregulatory functions of mesenchymal stem cells in patients with low-risk or high-risk myelodysplastic syndromes. PLoS One 2012; 7(9): e45675. doi: 10.1371/journal.pone.0045675

42. Phinney DG, Kopen G, Righter W, Webster S, Tremain N, Prockop DJ. Donor variation in the growth properties and osteogenic potential of human marrow stromal cells. J Cell Biochem 1999; 75(3): 424-36. doi: 10.1002/(SICI)10974644(19991201)75:3\%3C424::AID-JCB8\%3E3.0.CO;2-8

43. Phinney DG, Kopen G, Isaacson RL, Prockop DJ. Plastic adherent stromal cells from the bone marrow of commonly used strains of inbred mice: variations in yield, growth, and differentiation. J Cell Biochem 1999; 72(4): 570-85. doi: 10.1002/(SICI)10974644(19990315)72:4\%3C570::AID-JCB12\%3E3.0.CO;2-W

44. Zhou S, Greenberger JS, Epperly MW, Goff JP, Adler C, Leboff MS, et al. Age-related intrinsic changes in human bone-marrow-derived mesenchymal stem cells and their differentiation to osteoblasts. Aging Cell 2008; 7(3): 335-43. doi: 10.1111/j.1474-9726.2008.00377.x

45. Kang I, Lee BC, Choi SW, Lee JY, Kim JJ, Kim BE, et al. Donor-dependent variation of human umbilical cord blood mesenchymal stem cells in response to hypoxic preconditioning and amelioration of limb ischemia. Exp Mol Med 2018; 50(4): 1-15. doi: 10.1111/j.1474-9726.2008.00377.x

46. Cheng CC, Lee YH, Lin SP, Huangfu WC, Liu IH. Cellautonomous heparanase modulates self-renewal and migration in bone marrow-derived mesenchymal stem cells. J Biomed Sci 2014; 21(1): 21. doi: 10.1186/1423-0127-21-21

47. Wagner W, Ho AD. Mesenchymal stem cell preparations - comparing apples and oranges. Stem Cell Rev 2007; 3(4): 239-48. doi: 10.1007/s12015-007-9001-1

48. Hung SP, Ho JH, Shih YR, Lo T, Lee OK. Hypoxia promotes proliferation and osteogenic differentiation potentials of human mesenchymal stem cells. J Orthop Res 2012; 30(2): 260-6. doi: 10.1002/jor.21517

49. Fotia C, Massa A, Boriani F, Baldini N, Granchi D. Hypoxia enhances proliferation and stemness of human adipose-derived mesenchymal stem cells. Cytotechnology 2015; 67(6): 1073-84. doi: 10.1007/s10616-014-9731-2

50. Holzwarth C, Vaegler M, Gieseke F, Pfister SM, Handgretinger $\mathrm{R}$, Kerst $\mathrm{G}$, et al. Low physiologic oxygen tensions reduce proliferation and differentiation of human multipotent mesenchymal stromal cells. BMC Cell Biol 2010; 11: 11. doi: 10.1186/1471-2121-11-11

51. Wang Y, Wu H, Yang Z, Chi Y, Meng L, Mao A, et al. Human mesenchymal stem cells possess different biological characteristics but do not change their therapeutic potential when cultured in serum free medium. Stem Cell Res Ther 2014; 5(6): 132. doi: $10.1186 /$ scrt522

52. Ryan JM, Barry F, Murphy JM, Mahon BP. Interferon-gamma does not break, but promotes the immunosuppressive capacity of adult human mesenchymal stem cells. Clin Exp Immunol 2007; 149(2): 353-63. doi: 10.1111/j.1365-2249.2007.03422.x

53. Prasanna SJ, Gopalakrishnan D, Shankar SR, Vasandan AB. Pro-inflammatory cytokines, IFNgamma and TNFalpha, influence immune properties of human bone marrow and Wharton jelly mesenchymal stem cells differentially. PLoS One 2010; 5(2): e9016. doi: 10.1371/journal.pone.0009016

54. Cosgrove BD, Mui KL, Driscoll TP, Caliari SR, Mehta KD, Assoian RK, et al. N-cadherin adhesive interactions modulate matrix mechanosensing and fate commitment of mesenchymal stem cells. Nat Mater 2016; 15(12): 1297-306. doi: 10.1038/ nmat 4725

55. Cheng NC, Wang S, Young TH. The influence of spheroid formation of human adipose-derived stem cells on chitosan films on stemness and differentiation capabilities. Biomaterials 2012; 33(6): 1748-58. doi: 10.1016/j.biomaterials.2011.11.049

56. Araújo AB, Salton GD, Furlan JM, Schneider N, Angeli MH, Laureano Á M, et al. Comparison of human mesenchymal stromal cells from four neonatal tissues: amniotic membrane, chorionic membrane, placental decidua and umbilical cord. Cytotherapy 2017; 19(5): 577-85. doi: 10.1016/j.jcyt.2017.03.001

57. Stubbendorff M, Deuse T, Hua X, Phan TT, Bieback K, Atkinson $\mathrm{K}$, et al. Immunological properties of extraembryonic human mesenchymal stromal cells derived from gestational tissue. Stem Cells Dev 2013; 22(19): 2619-29. doi: 10.1089/ scd.2013.0043

58. Kern S, Eichler H, Stoeve J, Klüter H, Bieback K. Comparative analysis of mesenchymal stem cells from bone marrow, umbilical 
cord blood, or adipose tissue. Stem Cells 2006; 24(5): 1294-301. doi: 10.1634/stemcells.2005-0342

59. Hsiao ST, Asgari A, Lokmic Z, Sinclair R, Dusting GJ, Lim $\mathrm{SY}$, et al. Comparative analysis of paracrine factor expression in human adult mesenchymal stem cells derived from bone marrow, adipose, and dermal tissue. Stem Cells Dev 2012; 21(12): 2189-203. doi: 10.1089/scd.2011.0674

60. Han ZC, Du WJ, Han ZB, Liang L. New insights into the heterogeneity and functional diversity of human mesenchymal stem cells. Biomed Mater Eng 2017; 28(Suppl 1): S29-45. doi: 10.3233/BME-171622

61. Lu LL, Liu YJ, Yang SG, Zhao QJ, Wang X, Gong W, et al. Isolation and characterization of human umbilical cord mesenchymal stem cells with hematopoiesis-supportive function and other potentials. Haematologica 2006; 91(8): 1017-26. doi: $10.3324 / \% 25$

62. Baksh D, Yao R, Tuan RS. Comparison of proliferative and multilineage differentiation potential of human mesenchymal stem cells derived from umbilical cord and bone marrow. Stem Cells 2007; 25(6): 1384-92. doi: 10.1634/stemcells.2006-0709

63. Liu M, Yang SG, Shi L, Du WT, Liu PX, Xu J, et al. Mesenchymal stem cells from bone marrow show a stronger stimulating effect on megakaryocyte progenitor expansion than those from non-hematopoietic tissues. Platelets 2010; 21(3): 199-210. doi: 10.3109/09537101003602483

64. Salehinejad P, Alitheen NB, Ali AM, Omar AR, Mohit M, Janzamin E, et al. Comparison of different methods for the isolation of mesenchymal stem cells from human umbilical cord Wharton's jelly. In Vitro Cell Dev Biol Anim 2012; 48(2): 75-83. doi: 10.1007/s11626-011-9480-x

65. Hua J, Gong J, Meng H, Xu B, Yao L, Qian M, et al. Comparison of different methods for the isolation of mesenchymal stem cells from umbilical cord matrix: proliferation and multilineage differentiation as compared to mesenchymal stem cells from umbilical cord blood and bone marrow. Cell Biol Int 2014; 38(2): 198-210. doi: 10.1002/cbin. 10188

66. Horn P, Bork S, Diehlmann A, Walenda T, Eckstein V, Ho AD, et al. Isolation of human mesenchymal stromal cells is more efficient by red blood cell lysis. Cytotherapy 2008; 10(7): 676-85. doi: 10.1002/cbin. 10188

67. Horn P, Bokermann G, Cholewa D, Bork S, Walenda T, Koch $\mathrm{C}$, et al. Impact of individual platelet lysates on isolation and growth of human mesenchymal stromal cells. Cytotherapy 2010; 12(7): 888-98. doi: 10.3109/14653249.2010.501788

68. Hermida-Gómez T, Fuentes-Boquete I, Gimeno-Longas MJ, Muiños-López E, Díaz-Prado S, de Toro FJ, et al. Bone marrow cells immunomagnetically selected for CD271+ antigen promote in vitro the repair of articular cartilage defects. Tissue Eng Part A 2011; 17(7-8): 1169-79. doi: 10.1089/ten.tea.2010.0346

69. Yang ZX, Han ZB, Ji YR, Wang YW, Liang L, Chi Y, et al. CD106 identifies a subpopulation of mesenchymal stem cells with unique immunomodulatory properties. PLoS One 2013; 8(3): e59354. doi: 10.1089/ten.tea.2010.0346

70. Guérette D, Khan PA, Savard PE, Vincent M. Molecular evolution of type VI intermediate filament proteins. BMC Evol Biol 2007; 7: 164. doi: 10.1186/1471-2148-7-164

71. Yen BL, Huang HI, Chien CC, Jui HY, Ko BS, Yao M, et al. Isolation of multipotent cells from human term placenta. Stem Cells 2005; 23(1): 3-9. doi: 10.1634/stemcells.2004-0098

72. Wang W, Han ZC. Heterogeneity of human mesenchymal stromal/stem cells. Adv Exp Med Biol 2019; 1123: 165-77. doi: 10.1007/978-3-030-11096-3_10
73. Lv FJ, Tuan RS, Cheung KM, Leung VY. Concise review: the surface markers and identity of human mesenchymal stem cells. Stem Cells 2014; 32(6): 1408-19. doi: 10.1007/978-3-030-11096-3_10

74. Bensidhoum M, Chapel A, Francois S, Demarquay C, Mazurier C, Fouillard L, et al. Homing of in vitro expanded Stro-1- or Stro$1+$ human mesenchymal stem cells into the NOD/SCID mouse and their role in supporting human CD34 cell engraftment. Blood 2004; 103(9): 3313-19. doi: 10.1182/blood-2003-04-1121

75. Kuçi S, Kuçi Z, Kreyenberg H, Deak E, Pütsch K, Huenecke S, et al. CD271 antigen defines a subset of multipotent stromal cells with immunosuppressive and lymphohematopoietic engraftment-promoting properties. Haematologica 2010; 95(4): 651-9. doi: 10.3324/haematol.2009.015065

76. Sorrentino A, Ferracin M, Castelli G, Biffoni M, Tomaselli G, Baiocchi M, et al. Isolation and characterization of CD146+ multipotent mesenchymal stromal cells. Exp Hematol 2008; 36(8): 1035-46. doi: 10.3324/haematol.2009.015065

77. Mabuchi Y, Morikawa S, Harada S, Niibe K, Suzuki S, RenaultMihara F, et al. LNGFR(+)THY-1(+)VCAM-1(hi+) cells reveal functionally distinct subpopulations in mesenchymal stem cells. Stem Cell Rep 2013; 1(2): 152-65. doi: 10.1016/j. stemcr.2013.06.001

78. Fukiage K, Aoyama T, Shibata KR, Otsuka S, Furu M, Kohno Y, et al. Expression of vascular cell adhesion molecule-1 indicates the differentiation potential of human bone marrow stromal cells. Biochem Biophys Res Commun 2008; 365(3): 406-12. doi: 10.1016/j.bbrc.2007.10.149

79. Gang EJ, Bosnakovski D, Figueiredo CA, Visser JW, Perlingeiro RC. SSEA-4 identifies mesenchymal stem cells from bone marrow. Blood 2007; 109(4): 1743-51. doi: 10.1182/ blood-2005-11-010504

80. Zeddou M, Briquet A, Relic B, Josse C, Malaise MG, Gothot A, et al. The umbilical cord matrix is a better source of mesenchymal stem cells (MSC) than the umbilical cord blood. Cell Biol Int 2010; 34(7): 693-701. doi: 10.1042/CBI20090414

81. Wagner W, Wein F, Seckinger A, Frankhauser M, Wirkner U, Krause U, et al. Comparative characteristics of mesenchymal stem cells from human bone marrow, adipose tissue, and umbilical cord blood. Exp Hematol 2005; 33(11): 1402-16. doi: 10.1042/CBI20090414

82. Costa LA, Eiro N, Fraile M, Gonzalez LO, Saá J, GarciaPortabella P, et al. Functional heterogeneity of mesenchymal stem cells from natural niches to culture conditions: implications for further clinical uses. Cell Mol Life Sci 2021; 78(2): 447-67. doi: 10.1007/s00018-020-03600-0

83. Kebriaei P, Hayes J, Daly A, Uberti J, Marks DI, Soiffer R, et al. A phase 3 randomized study of remestemcel-L versus placebo added to second-line therapy in patients with steroid-refractory acute graft-versus-host disease. Biol Blood Marrow Transplant 2020; 26(5): 835-44. doi: 10.1016/j.bbmt.2019.08.029

84. Kuçi Z, Bönig H, Kreyenberg H, Bunos M, Jauch A, Janssen JW, et al. Mesenchymal stromal cells from pooled mononuclear cells of multiple bone marrow donors as rescue therapy in pediatric severe steroid-refractory graft-versus-host disease: a multicenter survey. Haematologica 2016; 101(8): 985-94. doi: 10.3324/ haematol.2015.140368

85. Sun C, Wang L, Wang H, Huang T, Yao W, Li J, et al. Single-cell RNA-seq highlights heterogeneity in human primary Wharton's jelly mesenchymal stem/stromal cells cultured in vitro. Stem Cell Res Ther 2020; 11(1): 149. doi: 10.3324/haematol.2015.140368

86. Hou W, Duan L, Huang C, Li X, Xu X, Qin P, et al. Mesenchymal stem cell subpopulations and their heterogeneity of response 
to inductions revealed by single-cell RNA-seq. bioRxiv 2021:2021.05.07.443197. doi: 10.1101/2021.05.07.443197

87. Huang Y, Li Q, Zhang K, Hu M, Wang Y, Du L, et al. Single cell transcriptomic analysis of human mesenchymal stem cells reveals limited heterogeneity. Cell Death Dis 2019; 10(5): 368. doi: 10.1038/s41419-019-1583-4

88. Zhou W, Lin J, Zhao K, Jin K, He Q, Hu Y, et al. Single-cell profiles and clinically useful properties of human mesenchymal stem cells of adipose and bone marrow origin. Am J Sports Med 2019; 47(7): 1722-33. doi: 10.1177/0363546519848678

89. Wang Y, Dai W, Liu Z, Liu J, Cheng J, Li Y, et al. Single-cell infrared microspectroscopy quantifies dynamic heterogeneity of mesenchymal stem cells during adipogenic differentiation. Anal Chem 2021; 93(2): 671-6. doi: 10.1021/acs.analchem.0c04110

90. Russell KC, Phinney DG, Lacey MR, Barrilleaux BL, Meyertholen KE, O'Connor KC. In vitro high-capacity assay to quantify the clonal heterogeneity in trilineage potential of mesenchymal stem cells reveals a complex hierarchy of lineage commitment. Stem Cells 2010; 28(4): 788-98. doi: 10.1002/ stem. 312

91. Rennerfeldt DA, Raminhos JS, Leff SM, Manning P, Van Vliet KJ. Emergent heterogeneity in putative mesenchymal stem cell colonies: single-cell time lapsed analysis. PLoS One 2019; 14(4): e0213452. doi: 10.1371/journal.pone.0213452

92. Mareddy S, Broadbent J, Crawford R, Xiao Y. Proteomic profiling of distinct clonal populations of bone marrow mesenchymal stem cells. J Cell Biochem 2009; 106(5): 776-86. doi: 10.1002/ jcb. 22088

93. Moravcikova E, Meyer EM, Corselli M, Donnenberg VS, Donnenberg AD. Proteomic profiling of native unpassaged and culture-expanded mesenchymal stromal cells (MSC). Cytometry A 2018; 93(9): 894-904. doi: 10.1002/cyto.a.23574

94. Yin L, Wu Y, Yang Z, Tee CA, Denslin V, Lai Z, et al. Microfluidic label-free selection of mesenchymal stem cell subpopulation during culture expansion extends the chondrogenic potential in vitro. Lab Chip 2018; 18(6): 878-89. doi: 10.1039/ C7LC01005B

95. Lam J, Marklein RA, Jimenez-Torres JA, Beebe DJ, Bauer SR, Sung KE. Adaptation of a simple microfluidic platform for high-dimensional quantitative morphological analysis of human mesenchymalstromalcells on polystyrene-based substrates. SLAS Technol 2017; 22(6): 646-61. doi: 10.1177/2472630317726050
96. Liu Z, Screven R, Yu D, Boxer L, Myers MJ, Han J, et al. Microfluidic separation of canine adipose-derived mesenchymal stromal cells. Tissue Eng Part C Methods 2021; 27(8): 445-61. doi: $10.1089 /$ ten.tec. 2021.0082

97. Bloor AJC, Patel A, Griffin JE, Gilleece MH, Radia R, Yeung DT, et al. Production, safety and efficacy of iPSC-derived mesenchymal stromal cells in acute steroid-resistant graft versus host disease: a phase I, multicenter, open-label, dose-escalation study. Nat Med 2020; 26(11): 1720-5. doi: 10.1038/s41591-020-1050-x

98. Ozay EI, Vijayaraghavan J, Gonzalez-Perez G, Shanthalingam S, Sherman HL, Garrigan DT, Jr., et al. Cymerus ${ }^{\mathrm{TM}}$ iPSC-MSCs significantly prolong survival in a pre-clinical, humanized mouse model of Graft-vs-host disease. Stem Cell Res 2019; 35: 101401. doi: 10.1016/j.scr.2019.101401

99. Hu X, Li L, Yu X, Zhang R, Yan S, Zeng Z, et al. CRISPR/ Cas9-mediated reversibly immortalized mouse bone marrow stromal stem cells (BMSCs) retain multipotent features of mesenchymal stem cells (MSCs). Oncotarget 2017; 8(67): 111847 65. doi: 10.18632/oncotarget.22915

100. Sarker SR, Aoshima Y, Hokama R, Inoue T, Sou K, Takeoka $\mathrm{S}$. Arginine-based cationic liposomes for efficient in vitro plasmid DNA delivery with low cytotoxicity. Int J Nanomed 2013; 8: 1361-75. doi: 10.2147/IJN.S38903

101.Zabaleta N, Barberia M, Martin-Higueras C, Zapata-Linares N, Betancor I, Rodriguez S, et al. CRISPR/Cas9-mediated glycolate oxidase disruption is an efficacious and safe treatment for primary hyperoxaluria type I. Nat Commun 2018; 9(1): 5454. doi: 10.1038/s41467-018-07827-1

102. Qiao Y, Xu Z, Yu Y, Hou S, Geng J, Xiao T, et al. Single cell derived spheres of umbilical cord mesenchymal stem cells enhance cell stemness properties, survival ability and therapeutic potential on liver failure. Biomaterials 2020; 227: 119573. doi: 10.1016/j.biomaterials.2019.119573

\section{*Guangli Suo}

CAS Key Laboratory of Nano-Bio Interface

Suzhou Institute of Nano-Tech and Nano-Bionics

Chinese Academy of Sciences

Suzhou, Jiangsu 215123, China

Email: glsuo2013@sinano.ac.cn 